\title{
An efficient multigrid solver for a reformulated version of the poroelasticity system is
}

\author{
F.J. Gaspar ${ }^{\text {a }}$, F.J. Lisbona ${ }^{\text {a }}$, C.W. Oosterlee ${ }^{\text {b,* }}$, P.N. Vabishchevich ${ }^{c}$ \\ ${ }^{a}$ Departamento de Mathemática Aplicada, University of Zaragoza, Pedro Cerbuna, 12, 50009 Zaragoza, Spain \\ ${ }^{\mathrm{b}}$ Delft University of Technology, Delft Institute of Applied Mathematics (DIAM), Mekelweg 4, 2628 CD Delft, The Netherlands \\ ${ }^{\mathrm{c}}$ Institute for Mathematical Modelling, Russian Academy of Sciences, 4-A, Miusskaya Sq., 124047 Moscow, Russia
}

Received 15 July 2005; received in revised form 9 March 2006; accepted 9 March 2006

\begin{abstract}
In this paper, we present a robust and efficient multigrid solver for a transformed version of the system of poroelasticity equations. The transformation enables us to treat the system in a decoupled fashion. We show that the transformation boils down to a stabilization term in the iterative scheme, and that the solution of the original problem is identical to the solution of the transformed problem. A highly efficient multigrid method can be developed, confirmed by numerical experiments.
\end{abstract}

(C) 2006 Elsevier B.V. All rights reserved.

Keywords: Poroelasticity; Transformation; Stability; Collocated grid; Multigrid

\section{Introduction}

In this paper, we would like to add an application and its efficient numerical treatment to the recently increased interest in saddle point type problems [2]. It is the time-dependent incompressible system of poroelasticity equations [3]. After a semi-discretization in time, the two-dimensional system, in its original form, can be written as a block $2 \times 2$ system,

$$
\left[\begin{array}{cc}
\mathscr{A} & \mathscr{G} \\
\mathscr{D} & -\tau \cdot \mathscr{B}
\end{array}\right]\left[\begin{array}{l}
\mathbf{u} \\
p
\end{array}\right]=\left[\begin{array}{l}
\mathbf{0} \\
f
\end{array}\right] \text {. }
$$

Here, $\mathbf{u}=(u, v)$ is the deformation (in the $x$ - and $y$-directions), $p$ is the pore pressure, $\mathscr{A}$ is the $2 \mathrm{D}$ linear elasticity operator, $\mathscr{D}$ is the divergence operator, $\mathscr{G}$ is the gradient, and $\mathscr{B}$ is a Laplace type operator multiplied by the time

\footnotetext{
This research has been partially supported by the INTAS project 03 50-4395, the Spanish project MEC/FEDER MTM 2004-019051 and the Diputación General de Aragón.

* Corresponding author.

E-mail address: c.w.oosterlee@math.tudelft.nl (C.W. Oosterlee).
}

step $\tau$. Source terms $f(x, t)$ are supposed to be in $L^{2}(\Omega)$. It represents a forced fluid extraction or injection process.

For very small time steps, the size of the lower diagonal block entry in (1) can become arbitrarily small. So, the system may be viewed as a sort of singularly perturbed system related to

$$
\left[\begin{array}{ll}
\mathscr{A} & \mathscr{G} \\
\mathscr{D} & O
\end{array}\right]\left[\begin{array}{l}
\mathbf{u} \\
p
\end{array}\right]=\left[\begin{array}{l}
\mathbf{0} \\
f
\end{array}\right] \text {. }
$$

Poroelasticity theory addresses the time dependent coupling between the deformation of porous material and the fluid flow inside. The porous matrix is supposed to be saturated by the fluid phase. The state of this continuous medium is characterized by the knowledge of elastic displacements and fluid pressure at each point. A phenomenological model was first proposed and analyzed by Biot [3], studying the consolidation of soils. Poroelastic models are used to study problems in geomechanics, hydrogeology, petrol engineering and biomechanics $[8,5]$.

In this paper, we present an efficient multigrid method for a transformed version of the system of poroelasticity 
equations. We show by means of block matrix manipulations that system (1) can be brought in a form which is favorable for (almost) decoupled iterative solution. At the same time, we analyze the transformed system and prove that the solution of this system is identical to the solution of the original system. We show in 1D that by the transformation, after discretization, a numerical stabilization term of lower order has been brought in the discrete version of the lower diagonal block of (1), which does not influence the order of numerical convergence negatively. By a onedimensional poroelasticity analysis and by corresponding numerical experiments we show the stabilizing effect of the term. Numerical 2D experiments confirm the stability, accuracy and the efficient multigrid treatment of the resulting system.

The paper is organized as follows, the poroelastic system, its transformation plus a corresponding solution algorithm are presented in Section 2. Furthermore, in Section 2.2 a previously used stable discretization on a staggered grid is explained, which is used for comparison with the current approach. In Section 3 the one-dimensional case is considered. The discrete problem is stabilized and the convergence of the corresponding scheme is proven. In a diagram the relation between the original system and the transformed system with respect to stability, both in the continuous case and in the discrete case is also presented. Section 4 then details the solution algorithm with only multigrid methods for scalar equations. Finally, Section 5 presents numerical poroelastic experiments indicating the efficiency of the solution algorithm.

\section{Mathematical model and discretization}

\subsection{Continuous system}

The quasi-static Biot model for soil consolidation can be formulated as a system of partial differential equations for displacements and the pressure of the fluid. One assumes the material's solid structure to be linearly elastic, initially homogeneous and isotropic, the strains imposed within the material are small. We denote by $\overline{\mathbf{u}}=(u, v, p)^{\mathrm{T}}$ the solution vector, consisting of the displacement vector $\mathbf{u}=(u, v)^{\mathrm{T}}$ and pore pressure of the fluid $p$. The governing equations read

$-\mu \tilde{\Delta} \mathbf{u}-(\lambda+\mu) \operatorname{grad} \operatorname{div} \mathbf{u}+\alpha \operatorname{grad} p=\mathbf{0}, \quad \mathbf{x} \in \Omega$,

$\alpha \frac{\partial}{\partial t}(\operatorname{div} \mathbf{u})-\frac{\kappa}{\eta} \Delta p=f(\mathbf{x}, t), \quad 0<t \leqslant T$,

where $\lambda$ and $\mu$ are the Lamé coefficients; $\kappa$ is the permeability of the porous medium, $\eta$ the viscosity of the fluid, $\alpha$ is the Biot-Willis constant (which we will take equal one) and $\tilde{\Delta}$ represents the vectorial Laplace operator. The quantity $\operatorname{div} \mathbf{u}(\mathbf{x}, t)$ is the dilatation, i.e. the volume increase rate of the system, a measure of the change in porosity of the soil. The source term $f(\mathbf{x}, t)$ represents a forced fluid extraction or injection process, respectively, see [3].
For simplicity, we assume here that $\partial \Omega$ is rigid (zero displacements) and permeable (free drainage), so that we have homogeneous Dirichlet boundary conditions,

$\mathbf{u}(\mathbf{x}, t)=0, \quad p(\mathbf{x}, t)=0, \quad \mathbf{x} \in \partial \Omega$.

Before fluid starts to flow and due to the incompressibility of the solid and fluid phases, the initial state satisfies

$\operatorname{div} \mathbf{u}(\mathbf{x}, 0)=0, \quad \mathbf{x} \in \Omega$.

In the numerical experiments in Section 5 more complicated boundary conditions are chosen. The incompressible, two-dimensional variant of Biot's consolidation model reads

$$
\begin{aligned}
& -(\lambda+2 \mu) u_{x x}-\mu u_{y y}-(\lambda+\mu) v_{x y}+p_{x}=0, \\
& -(\lambda+\mu) u_{x y}-\mu v_{x x}-(\lambda+2 \mu) v_{y y}+p_{y}=0, \\
& \left(u_{x}+v_{y}\right)_{t}-a\left(p_{x x}+p_{y y}\right)=f
\end{aligned}
$$

(plus initial and boundary conditions) with $a=\kappa / \eta>0$. Problem (7) is a limit of the compressible case. The compressible system is, however, easier to solve due to an extra contribution to the main diagonal of the matrix related to this system. We concentrate on a solver for the two-dimensional incompressible case, and consider a model operator $\mathbf{L}$, which reads

$$
\mathbf{L}=\left(\begin{array}{ccc}
-(\lambda+2 \mu) \partial_{x x}-\mu \partial_{y y} & -(\lambda+\mu) \partial_{x y} & \partial_{x} \\
-(\lambda+\mu) \partial_{x y} & -\mu \partial_{x x}-(\lambda+2 \mu) \partial_{y y} & \partial_{y} \\
\partial_{x} & \partial_{y} & -\tilde{a}\left(\partial_{x x}+\partial_{y y}\right)
\end{array}\right) .
$$

$\mathbf{L}$ can be interpreted as a "stationary variant" of (7), i.e., the operator after an implicit (semi-) discretization in time. For example, in case of Crank-Nicholson time discretization we have $\tilde{a}=0.5 a \tau$. From (8) one may calculate the corresponding determinant:

$\operatorname{det}(\mathbf{L})=-\mu \Delta\left(\tilde{a}(\lambda+2 \mu) \Delta^{2}-\Delta\right)$

with Laplace operator $\Delta$ and biharmonic operator $\Delta^{2}$. The principal part of $\operatorname{det}(\mathbf{L})$ is $\Delta^{m}$ with $m$ depending on the choice of $\lambda, \mu$, and $\tilde{a}$. From physical reasoning, we always have $\mu, \tilde{a}, \lambda+2 \mu>0$, yielding $m=3$. The number of boundary conditions that must accompany $\mathbf{L}$ is $m[4,12]$.

\subsection{Previous approach: staggered grid}

Physically, when a load is applied in a poroelasticity problem, the pressure suddenly increases and a boundary layer appears in the early stages of the time-dependent process. In the case of an unstable discretization, unphysical oscillations can occur in the first time steps of the solution. After this phase, the solution shows a much smoother behavior. The time-dependent operator (8) suffers from stability difficulties. The coefficient in the $L^{3,3}$-block in (8) is typically, depending on the time step, extremely small. In order to avoid oscillating solutions, the discretization has to be designed with care.

Previously, we adopted a staggered grid discretization in [6] for system (7), using nearest neighbor central finite dif- 
ferences. Pressure points in the staggered grid were located at the physical boundary, and the displacement points are defined at the cell faces, as often pressure is prescribed at the boundary. The divergence operator is naturally approximated by a central discretization of the displacements in a cell, see Fig. 1.

The discretization of each equation, centered around the equation's primary unknown, reads in this case

$\mathbf{L}_{h} \overline{\mathbf{u}}_{h}=\left(\begin{array}{ccc}-(\lambda+\mu)\left(\partial_{x x}\right)_{h}-\mu \Delta_{h} & -(\lambda+\mu)\left(\partial_{x y}\right)_{h} & \left(\partial_{x}\right)_{h} \\ -(\lambda+\mu)\left(\partial_{x y}\right)_{h} & -\mu \Delta_{h}-(\lambda+\mu)\left(\partial_{y y}\right)_{h} & \left(\partial_{y}\right)_{h} \\ \left(\partial_{x}\right)_{h} & \left(\partial_{y}\right)_{h} & -\tilde{a} \Delta_{h}\end{array}\right)\left(\begin{array}{c}u_{h} \\ v_{h} \\ p_{h}\end{array}\right)=\mathbf{f}_{h}$,

with $\mathbf{f}_{h}=\left(0,0, f_{h}\right)^{\mathrm{T}}$, and the subscripts denote central discrete operators on the staggered grid. An efficient multigrid solver for the system of poroelasticity equations discretized on the staggered grid has been developed in $[7,13]$.

The multigrid scheme developed solves the system in a coupled fashion, with a distributive smoother: In order to relax $\mathbf{L}_{h} \overline{\mathbf{u}}_{h}=\mathbf{f}_{h}$, a new variable $\mathbf{w}_{h}$ by $\overline{\mathbf{u}}_{h}=\mathbf{C}_{h} \mathbf{w}_{h}$ was introduced and system $\mathbf{L}_{h} \mathbf{C}_{h} \mathbf{w}_{h}=\mathbf{f}_{h}$ has been considered in smoothing. The resulting system is suited for decoupled smoothing, i.e., each equation can be treated separately. The so-called distributor reads [13]

$$
\mathbf{C}_{h}=\left(\begin{array}{ccc}
I_{h} & 0 & -\left(\partial_{x}\right)_{h} \\
0 & I_{h} & -\left(\partial_{y}\right)_{h} \\
(\lambda+\mu)\left(\partial_{x}\right)_{h} & (\lambda+\mu)\left(\partial_{y}\right)_{h} & -(\lambda+2 \mu) \Delta_{h}
\end{array}\right)
$$

with identity $I_{h}$. Then, the system reads

$$
\mathbf{L}_{h} \mathbf{C}_{h}=\left(\begin{array}{ccc}
-\mu \Delta_{h} & 0 & 0 \\
0 & -\mu \Delta_{h} & 0 \\
L C_{h}^{3,1} & L C_{h}^{3,2} & \tilde{a}(\lambda+2 \mu) \Delta_{h}^{2}-\Delta_{h}
\end{array}\right)
$$

with

$L C_{h}^{3,1}=\left(\partial_{x}\right)_{h}-\tilde{a}(\lambda+\mu)\left(\left(\partial_{x x x}\right)_{h}+\left(\partial_{x y y}\right)_{h}\right)$,

$L C_{h}^{3,2}=\left(\partial_{y}\right)_{h}-\tilde{a}(\lambda+\mu)\left(\left(\partial_{x x y}\right)_{h}+\left(\partial_{y y y}\right)_{h}\right)$.

Equation-wise smoothing for (11) is now an option, starting with the first equation in the system, etc. All multigrid components related to the coarse grid correction are naturally dictated by the staggered grid arrangement. The multigrid scheme works very well, with convergence factors less than $0.2[7,13]$.

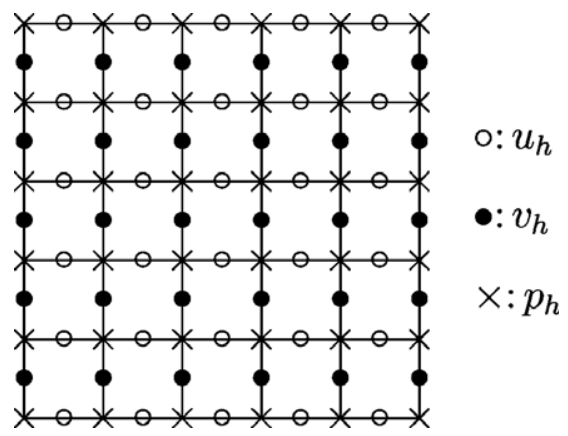

Fig. 1. Staggered location of unknowns for poroelasticity.
However, the choice for a staggered grid is a rigorous one. Staggered grid discretizations may not be easily generalized to curved domains, or to unstructured grids. The staggered grid choice has only been made, because in the initial phase of a time-dependent problem a boundary layer may occur that is prone to unphysical oscillations on a collocated grid, without additional stabilization. Therefore, we perform here a system transformation and (implicitly, by doing so) a stabilization so that we can discretize on a collocated grid, without any unphysical oscillations.

\subsection{Transformed system}

Let us rewrite problem (3)-(6) as

$A \frac{\partial \mathbf{u}}{\partial t}+\operatorname{grad} \frac{\partial p}{\partial t}=\mathbf{0}, \quad$ in $\Omega$,

$\operatorname{div} \frac{\partial \mathbf{u}}{\partial t}-\frac{\kappa}{\eta} \Delta p=f, \quad$ in $\Omega$,

$\mathbf{u}=\mathbf{0}, \quad p=0, \quad$ on $\partial \Omega$,

$\operatorname{div} \mathbf{u}(\mathbf{x}, 0)=0, \quad \mathbf{x} \in \Omega$,

where $A=-\mu \tilde{\Delta}-(\lambda+\mu) \operatorname{grad} \operatorname{div}$.

We transform problem (14)-(17) to an equivalent problem. Firstly, applying the divergence operator to (14) and the operator $(\lambda+2 \mu) \Delta$ to (15), adding the resulting equations and taking into account the equality

$-(\lambda+2 \mu) \Delta \operatorname{div}=\operatorname{div} A$,

we obtain

$-\Delta \frac{\partial p}{\partial t}+(\lambda+2 \mu) \frac{\kappa}{\eta} \Delta^{2} p=-(\lambda+2 \mu) \Delta f$.

Secondly, by applying operator $(\lambda+\mu)$ grad to (15) and by adding the resulting equation to (14) we get

$-\mu \tilde{\Delta} \frac{\partial \mathbf{u}}{\partial t}+\operatorname{grad} \frac{\partial p}{\partial t}-(\lambda+\mu) \frac{\kappa}{\eta} \operatorname{grad} \Delta p=(\lambda+\mu) \operatorname{grad} f$.

With the new variables $q=-\Delta p$ and $\mathbf{v}=\frac{\partial \mathbf{u}}{\partial t}$, we deal with the system:

$-\mu \tilde{\Delta} \mathbf{v}+\operatorname{grad} \frac{\partial p}{\partial t}+(\lambda+\mu) \frac{\kappa}{\eta} \operatorname{grad} q=(\lambda+\mu) \operatorname{grad} f$,

$q+\Delta p=0$

$\frac{\partial q}{\partial t}-(\lambda+2 \mu) \frac{\kappa}{\eta} \Delta q=-(\lambda+2 \mu) \Delta f$,

$\mathbf{v}=\mathbf{0}, \quad p=0, \quad \operatorname{div} \mathbf{v}+\frac{\kappa}{\eta} q=f, \quad$ on $\partial \Omega$

plus initial conditions. We have proven the following result:

Proposition 1. If ( $\mathbf{u}, p)$ is a solution of problem (14)-(17), then $(\mathbf{v}, p, q)$ is the solution of problem (19)-(22).

In the following proposition we prove that both problems really are equivalent.

Proposition 2. If $(\mathbf{v}, p, q)$ is solution of problem (19)-(22), then $(\mathbf{u}, p)$ is solution of problem (14)-(17). 
Proof. By applying the divergence operator to (19) and the use of equality $\operatorname{div} \tilde{\Delta}=\Delta \operatorname{div}$, we find

$-\mu \Delta \operatorname{div} \mathbf{v}-\frac{\partial q}{\partial t}+(\lambda+\mu) \frac{\kappa}{\eta} \Delta q=(\lambda+\mu) \Delta f$.

Adding (23) and (21), we obtain

$\mu \Delta\left(\operatorname{div} \mathbf{v}+\frac{\kappa}{\eta} q-f\right)=0, \quad$ in $\Omega$.

By using boundary conditions (22) we deduce Eq. (15). Eq.

(14) is obtained by applying the operator $(\lambda+\mu) \operatorname{grad}$ to (15) and using (19).

Note that problem (19)-(22) is coupled over the boundary of the domain.

A generalization of the transformation to three dimensions is straightforward. One additional unknown, the time-dependent displacement in the $z$-direction, is then also resolved.

Let us consider a semi-discretization in time with time step $\tau=T / M$ with $M$ a positive integer. For $1 \leqslant m \leqslant$ $M-1$ and assuming that $\mathbf{v}^{m}, p^{m}$ and $q^{m}$ are known, the following iterative scheme is proposed.

\section{Algorithm I}

(1) Solve:

$$
\left\{\begin{array}{cl}
\left(\frac{q^{m+1}-q^{m}}{\tau}\right)-(\lambda+2 \mu) \frac{\kappa}{\eta} \Delta q^{m+1} & \\
=-(\lambda+2 \mu) \Delta f^{m+1}, & \text { in } \Omega, \\
\operatorname{div} \mathbf{v}^{m}+\frac{\kappa}{\eta} q^{m+1}=f^{m+1} & \text { on } \partial \Omega .
\end{array}\right.
$$

(2) Solve

$$
\begin{cases}-\Delta p^{m+1}=q^{m+1} & \text { in } \Omega \\ p^{m+1}=0, & \text { on } \partial \Omega\end{cases}
$$

(3) Solve

$$
\left\{\begin{array}{cc}
-\mu \tilde{\Delta} \mathbf{v}^{m+1}+\operatorname{grad} \frac{p^{m+1}-p^{m}}{\tau} & \\
+(\lambda+\mu) \frac{\kappa}{\eta} \operatorname{grad} q^{m+1} & \\
=(\lambda+\mu) \operatorname{grad} f^{m+1}, & \text { in } \Omega, \\
\mathbf{v}^{m+1}=\mathbf{0}, & \text { on } \partial \Omega .
\end{array}\right.
$$

Notice that the boundary condition in step (1) is lagging behind one time step. Without additional iteration the scheme presented here will therefore be of $\mathcal{O}(\tau)$.

Two important issues regarding Algorithm I are discussed in Sections 3.1 and 4.

First of all, the operators to be inverted in the algorithm above are only scalar Laplace type operators, for which standard multigrid for scalar equations works extremely well (Section 4). Therefore, a highly efficient iterative solution process can be defined for the transformed system.
Secondly, when working with the transformed system stable numerical solutions are obtained on a standard collocated grid (Section 3.1). So far, we can prove this stability issue only for the 1D poroelasticity model situation.

\section{One-dimensional poroelasticity}

\subsection{D Transformation}

In $1 \mathrm{D}$, the governing equations simplify and read

$$
\begin{aligned}
(\mathbf{P}): \quad & -(\lambda+2 \mu) \frac{\partial^{2} u}{\partial x^{2}}+\frac{\partial p}{\partial x}=0, \\
& \frac{\partial^{2} u}{\partial x \partial t}-\frac{\kappa}{\eta} \frac{\partial^{2} p}{\partial x^{2}}=f, \quad x \in(0,1), 0<t \leqslant T,
\end{aligned}
$$

We denote the original problem (24) and (25) by $(\boldsymbol{P})$ for reference in Fig. 2. For simplicity, we again assume that $\partial \Omega$ is rigid (zero displacements) and permeable (free drainage), so that we have homogeneous Dirichlet boundary conditions, $u(0, t)=u(1, t)=0, p(0, t)=p(1, t)=0$, and the initial state satisfies

$\frac{\partial u}{\partial x}(x, 0)=0$

The equivalent transformed 1D problem, denoted by $\left(\mathbf{P}^{\mathrm{tr}}\right)$, is

$$
\begin{aligned}
\left(\mathbf{P}^{\mathrm{tr}}\right): & -(\lambda+2 \mu) \frac{\partial^{2} u}{\partial x^{2}}+\frac{\partial p}{\partial x}=0, \\
& q+\frac{\partial^{2} p}{\partial x^{2}}=0, \\
& \frac{\partial q}{\partial t}-(\lambda+2 \mu) \frac{\kappa}{\eta} \frac{\partial^{2} q}{\partial x^{2}}=-(\lambda+2 \mu) \frac{\partial^{2} f}{\partial x^{2}}, \\
& u=0, \quad p=0, \quad \frac{\partial^{2} u}{\partial x \partial t}+\frac{\kappa}{\eta} q=f, \quad \text { on } \partial \Omega .
\end{aligned}
$$

The 1D transformation is easier than the $2 \mathrm{D} / 3 \mathrm{D}$ cases, as it is not necessary to cancel out the term graddiv $u$ from the first equation.

\subsection{Discrete case}

We consider a uniform grid on the interval $[0,1]$, with step size $h$ :

$\bar{\omega}=\left\{x_{i} \mid x_{i}=i h, i=0,1 \ldots, N\right\}$,

and denote by $\omega$ and $\partial \omega$ the interior and the boundary nodes, respectively. We consider the Hilbert space $H_{\bar{\omega}}$ of

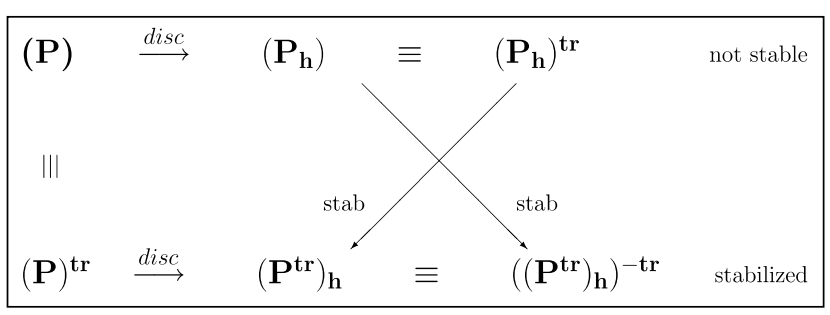

Fig. 2. Relation between stable and unstable, continuous and discrete problem formulations. 
the discrete functions $u_{h}=\left(u_{0}, u_{1}, \ldots, u_{N}\right)$ on the grid $\bar{\omega}$, with scalar product and norm given by

$\left(u_{h}, v_{h}\right)_{\bar{\omega}}=h\left(\frac{u_{0} v_{0}+u_{N} v_{N}}{2}+\sum_{i=1}^{N-1} u_{i} v_{i}\right), \quad\left\|u_{h}\right\|_{\bar{\omega}}=\sqrt{\left(u_{h}, u_{h}\right)}$.

In a similar way, let us consider the Hilbert space $H_{\omega}=$ $\left\{u_{h} \in H_{\bar{\omega}} \mid u_{0}=u_{N}=0\right\}$, with scalar product

$\left(u_{h}, v_{h}\right)=h \sum_{i=1}^{N-1} u_{i} v_{i}$,

and associated norm.

In order to handle the boundary conditions we introduce exterior nodes, called ghost points, constructed by symmetry with respect to the boundaries of the domain.

We define the self-adjoint and positive definite operator $\delta$ on $H_{\omega}$ as

$$
\left(\delta w_{h}\right)_{i}=-\frac{w_{i+1}-2 w_{i}+w_{i-1}}{h^{2}} \quad i=0, \ldots, N,
$$

and we consider the operators $A=(\lambda+2 \mu) \delta$ and $B=\delta$. We also introduce the difference operators, $G=D: H_{\omega} \rightarrow$ $H_{\bar{\omega}}$

$$
\left(G p_{h}\right)_{i}=\frac{p_{i+1}-p_{i-1}}{2 h}, \quad i=0, \ldots, N
$$

which verify $\left(G p_{h}, u_{h}\right)=-\left(p_{h}, D u_{h}\right), \forall\left(u_{h}, p_{h}\right) \in H_{\omega} \times H_{\omega}$.

Let $u_{h}^{m} \in H_{\omega}$ and $p_{h}^{m} \in H_{\omega}$ be approximations to $u\left(x, t_{m}\right)$ and $p\left(x, t_{m}\right)$, where $t_{m}=m \tau, \quad m=0,1, \ldots, M, \quad M \tau=T$. Then, using an Euler implicit scheme to discretize in time we get the problem $\left(\mathbf{P}_{\mathbf{h}}\right)$ :

$$
\begin{aligned}
\left(\mathbf{P}_{\mathbf{h}}\right): & A u_{h}^{m+1}+G p_{h}^{m+1}=0, \\
& \frac{D u_{h}^{m+1}-D u_{h}^{m}}{\tau}+\frac{\kappa}{\eta} B p_{h}^{m+1}=f_{h}^{m+1}, \\
& m=0, \ldots, M-1
\end{aligned}
$$

with initial condition $D u_{h}^{0}=0$. $\left(\mathbf{P}_{\mathbf{h}}\right)$ represents the discretization of the original problem on a collocated grid, which is not stable. If we follow the transformations made in the continuous case, i.e. apply operator $A$ to the second equation and operator $D$ to the first equation and take into account the initial condition $D u_{h}^{0}=0$, we obtain the transformed discrete problem, denoted by $\left(\mathbf{P}_{\mathbf{h}}\right)^{\text {tr }}$,

$$
\begin{aligned}
\left(\mathbf{P}_{\mathbf{h}}\right)^{\text {tr }}: & A u_{h}^{1}+G p_{h}^{1}=0, \\
& q_{h}^{1}=B p_{h}^{1}, \\
& C q_{h}^{1}+\tau(\lambda+2 \mu) \frac{\kappa}{\eta} B q_{h}^{1}=\tau(\lambda+2 \mu) B f_{h}^{1}, \\
& D\left(\frac{u_{h}^{1}}{\tau}\right)+\frac{\kappa}{\eta} q_{h}^{1}=f_{h}^{1}, \quad \text { on } \partial \bar{\omega},
\end{aligned}
$$

and

$$
\begin{aligned}
& A u_{h}^{m+1}+G p_{h}^{m+1}=0, \\
& q_{h}^{m+1}=B p_{h}^{m+1},
\end{aligned}
$$

$$
\begin{aligned}
& C\left(\frac{q_{h}^{m+1}-q_{h}^{m}}{\tau}\right)+(\lambda+2 \mu) \frac{\kappa}{\eta} B q_{h}^{m+1}=(\lambda+2 \mu) B f_{h}^{m+1}, \\
& D\left(\frac{u_{h}^{m+1}-u_{h}^{m}}{\tau}\right)+\frac{\kappa}{\eta} q_{h}^{m+1}=f_{h}^{m+1} \\
& \quad \text { on } \partial \bar{\omega}, m=1,2, \ldots, M-1,
\end{aligned}
$$

where a new difference operator appears, given by $\left(C q_{h}\right)_{i}=\frac{1}{4}\left(q_{i+1}+2 q_{i}+q_{i-1}\right)$. One can easily verify that $-D G=C B=B C$. In this way we have obtained a problem that is equivalent to problem (31) and (32).

Remark 1. With this scheme we may obtain oscillations because the coefficient matrices in systems (35) and (39) are, in general, no M-matrices. If $h^{2}<\frac{4(\lambda+2 \mu) \kappa}{\eta} \tau$ we have Mmatrices. However, this restriction on the spatial step is severe if we use small time steps in the initial stage of the consolidation process.

In order to stabilize Eqs. (35) and (39) we perturb them as follows:

$$
\begin{aligned}
& C q_{h}^{1}+\tau(\lambda+2 \mu) \frac{\kappa}{\eta} B q_{h}^{1}+\frac{h^{2}}{4} B q_{h}^{1}=\tau(\lambda+2 \mu) B f_{h}^{1}, \\
& C\left(\frac{q_{h}^{m+1}-q_{h}^{m}}{\tau}\right)+(\lambda+2 \mu) \frac{\kappa}{\eta} B q_{h}^{m+1}+\frac{h^{2}}{4} B\left(\frac{q_{h}^{m+1}-q_{h}^{m}}{\tau}\right) \\
& \quad=(\lambda+2 \mu) B f_{h}^{m+1} .
\end{aligned}
$$

For convenience, to obtain later a simple equivalent scheme, we also perturb the equations on the boundary in the appropriate way.

Taking into account that $C+\frac{h^{2}}{4} B=I$, we encounter the discrete problem

$$
\begin{aligned}
\left(\mathbf{P}^{\mathrm{tr}}\right)_{h}: & A u_{h}^{1}+G p_{h}^{1}=0, \\
& q_{h}^{1}=B p_{h}^{1}, \\
& q_{h}^{1}+\tau(\lambda+2 \mu) \frac{\kappa}{\eta} B q_{h}^{1}=\tau(\lambda+2 \mu) B f_{h}^{1}, \\
& D\left(\frac{u_{h}^{1}}{\tau}\right)+\frac{\kappa}{\eta} q_{h}^{1}+\frac{h^{2}}{4(\lambda+2 \mu)} q_{h}^{1}=f_{h}^{1}, \quad \text { on } \partial \bar{\omega},
\end{aligned}
$$

and

$$
\begin{aligned}
& A u_{h}^{m+1}+G p_{h}^{m+1}=0 \\
& q_{h}^{m+1}=B p_{h}^{m+1} \\
& \left(\frac{q_{h}^{m+1}-q_{h}^{m}}{\tau}\right)+(\lambda+2 \mu) \frac{\kappa}{\eta} B q_{h}^{m+1}=(\lambda+2 \mu) B f_{h}^{m+1} \\
& D\left(\frac{u_{h}^{m+1}-u_{h}^{m}}{\tau}\right)+\frac{\kappa}{\eta} q_{h}^{m+1}+\frac{h^{2}}{4(\lambda+2 \mu)}\left(\frac{q_{h}^{m+1}-q_{h}^{m}}{\tau}\right)=f_{h}^{m+1}, \\
& \quad \text { on } \partial \bar{\omega}, m=1,2, \ldots, M-1,
\end{aligned}
$$

which is a new discretization of problem (27)-(30), denoted by $\left(\mathbf{P}^{\mathbf{t r}}\right)_{h}$ (i.e., first transform then discretize). Finally, we observe that this formulation is equivalent to the pre-transformed scheme 


$$
\begin{aligned}
\left(\left(\mathbf{P}^{\mathrm{tr}}\right)_{h}\right)^{-t r}: \quad & A u_{h}^{1}+G p_{h}^{1}=0, \\
& D u_{h}^{1}+\tau \frac{\kappa}{\eta} B p_{h}^{1}+\frac{h^{2}}{4(\lambda+2 \mu)} B p_{h}^{1}=\tau f_{h}^{1},
\end{aligned}
$$

and

$$
\begin{aligned}
& A u_{h}^{m+1}+G p_{h}^{m+1}=0, \\
& \frac{D u_{h}^{m+1}-D u_{h}^{m}}{\tau}+\frac{\kappa}{\eta} B p_{h}^{m+1}+\frac{h^{2}}{4(\lambda+2 \mu)} \frac{B p_{h}^{m+1}-B p_{h}^{m}}{\tau}=f_{h}^{m+1}, \\
& \quad m=1,2, \ldots, M-1,
\end{aligned}
$$

where the initial condition $D u_{h}^{0}$ has already been applied. This scheme does not give oscillations. A diagram in Fig. 2 summarizes the relation between the different problems considered. In the figure disc stands for "discretized", stab means "stabilized" and $\equiv$ indicates that problems are equivalent. Summarizing, we have six different problems:

- $(\mathbf{P})$ : original continuous problem,

- $\left(\mathbf{P}^{\mathbf{t r}}\right)$ : transformed continuous problem,

- $\left(\mathbf{P}_{\mathbf{h}}\right)$ : discretization of (P) (this scheme shows oscillations),

- $\left(\mathbf{P}^{\mathbf{t r}}\right)_{\mathbf{h}}$ : discretization of $\left(\mathbf{P}^{\mathbf{t r}}\right)$ (oscillation-free solutions),

- $\left(\mathbf{P}_{\mathbf{h}}\right)^{\text {tr. }}$ : transformation of discrete problem $\left(\mathbf{P}_{\mathbf{h}}\right)$ identical to $\left(\mathbf{P}_{\mathbf{h}}\right)$ (i.e., oscillatory), and

- $\left(\left(\mathbf{P}^{\mathbf{t r}}\right)_{\mathbf{h}}\right)^{-\mathbf{t r}}$ : formulation where $\left(\mathbf{P}^{\mathbf{t r}}\right)_{\mathbf{h}}$ is transformed to an identical (stable) scheme based on the original problem.

Fig. 2 indicates that one can obtain $\left(\left(\mathbf{P}^{\text {tr }}\right)_{\mathbf{h}}\right)^{- \text {tr }}$ from $\left(\mathbf{P}_{\mathbf{h}}\right)$ by adding a stabilization term. We can also obtain scheme $\left(\mathbf{P}^{\mathbf{t r}}\right)_{\mathbf{h}}$ from $\left(\mathbf{P}_{\mathbf{h}}\right)^{\text {tr }}$ by adding a stabilization term.

In order to illustrate with a numerical example the effects of stabilization of term $h^{2} B\left(p_{h}^{m+1}-p_{h}^{m}\right) /(4(\lambda+2 \mu) \tau)$ as in (52), we show the numerical results for Terzagui problem [3], which consists on a column of soil, bounded by a rigid, impermeable bottom and walls. A unit load is prescribed at the top wall, which is free to drain.

Fig. 3 shows, for the initial time step, that standard central finite differences on a grid with 32 nodes lead to spurious oscillations in the discrete pressure $(x=0$ represents the top wall). The time discretization is the $\mathrm{O}(\tau)$ accurate implicit Euler scheme with $\tau=10^{-6}$.

In this problem the boundary layer is approximated in a stable way by the discrete transformed system in $\mathrm{O}\left(h^{2}+\tau\right)$ accuracy on a collocated grid with 32 nodes, see also Fig. 3.

\subsection{Convergence of the stabilized scheme}

We now prove in 1D that the stabilized discrete problem gives stable solutions. Firstly, we give an energy estimate. For simplicity, we suppose that $p_{h}^{0}$ satisfies the boundary conditions. In this case, the scheme writes as (51), (52) even for $m=0$. Otherwise, a different estimate must be done for the first step time.

Proposition 3. The solutions of the scheme (51) and (52) for $m \geqslant 0$ satisfy the a priori estimates

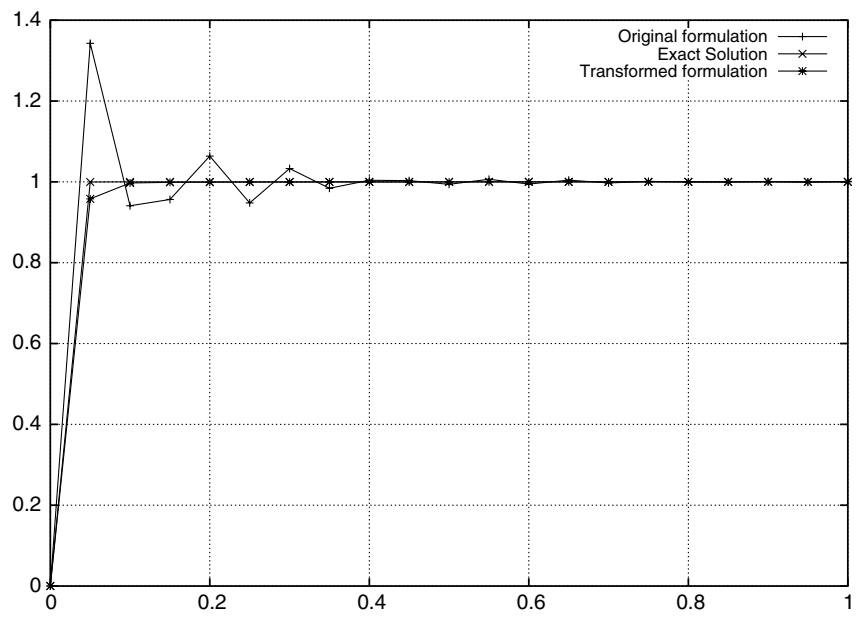

Fig. 3. Unphysical oscillations for original formulation of the incompressible poroelasticity model problem, with central differencing with 32 nodes, $\hat{t}=10^{-6}$, and stable solution from transformed system on a collocated grid.

$$
\begin{aligned}
& \left\|u_{h}^{m+1}\right\|_{A}^{2}+\frac{h^{2}}{4(\lambda+2 \mu)}\left\|p_{h}^{m+1}\right\|_{B}^{2} \\
& \leqslant\left\|u_{h}^{0}\right\|_{A}^{2}+\frac{h^{2}}{4(\lambda+2 \mu)}\left\|p_{h}^{0}\right\|_{B}^{2}+C_{1} \tau \sum_{j=1}^{m+1}\left\|f_{h}^{j}\right\|_{B^{-1}}^{2}, \\
& \left\|p_{h}^{m+1}\right\|_{B}^{2} \leqslant 2\left\|p_{h}^{0}\right\|_{B}^{2}+C_{2}\left(\tau \sum_{j=1}^{m}\left\|\frac{f_{h}^{j+1}-f_{h}^{j}}{\tau}\right\|_{B^{-1}}^{2}+\left\|f_{h}^{m+1}\right\|_{B^{-1}}^{2}\right),
\end{aligned}
$$

where $C_{1}$ and $C_{2}$ are constants independent of the discretization parameters.

Proof. Multiplying scalarly (51) and (52) by $\left(u_{h}^{m+1}-u_{h}^{m}\right) / \tau$ and $p_{h}^{m+1}$ respectively, we get for $0 \leqslant m \leqslant M-1$,

$$
\left(A u_{h}^{m+1}, \frac{u_{h}^{m+1}-u_{h}^{m}}{\tau}\right)+\left(G p_{h}^{m+1}, \frac{u_{h}^{m+1}-u_{h}^{m}}{\tau}\right)=0,
$$

and

$$
\begin{aligned}
& \left(D \frac{u_{h}^{m+1}-u_{h}^{m}}{\tau}, p_{h}^{m+1}\right)+\frac{\kappa}{\eta}\left(B p_{h}^{m+1}, p_{h}^{m+1}\right) \\
& +\frac{h^{2}}{4(\lambda+2 \mu)}\left(B \frac{p_{h}^{m+1}-p_{h}^{m}}{\tau}, p_{h}^{m+1}\right) \\
& =\left(f_{h}^{m+1}, p_{h}^{m+1}\right) .
\end{aligned}
$$

The addition of (55) and (56) yields

$$
\begin{aligned}
& \left(A u_{h}^{m+1}, u_{h}^{m+1}-u_{h}^{m}\right)+\tau \frac{\kappa}{\eta} B\left(p_{h}^{m+1}, p_{h}^{m+1}\right) \\
& \quad+\frac{h^{2}}{4(\lambda+2 \mu)}\left(B\left(p_{h}^{m+1}-p_{h}^{m}\right), p_{h}^{m+1}\right) \\
& \quad=\tau\left(f_{h}^{m+1}, p_{h}^{m+1}\right) .
\end{aligned}
$$

Applying the generalized Cauchy-Schwarz inequality in the right-hand side we get 


$$
\begin{aligned}
& \frac{1}{2}\left(\left\|u_{h}^{m+1}\right\|_{A}^{2}-\left\|u_{h}^{m}\right\|_{A}^{2}\right)+\frac{h^{2}}{8(\lambda+2 \mu)}\left(\left\|p_{h}^{m+1}\right\|_{B}^{2}-\left\|p_{h}^{m}\right\|_{B}^{2}\right) \\
& \quad \leqslant \frac{\tau \eta}{4 \kappa}\left\|f_{h}^{m+1}\right\|_{B^{-1}}^{2}
\end{aligned}
$$

and therefore, (53) is obtained, i.e., the solution $u_{h}$ is stable with respect to the initial data and right-hand side.

To obtain an a priori estimate for the pressure, a splitting of the solution will be used. Let be $p_{h}^{m+1}=$ $\bar{p}_{h}^{m+1}+\overline{\bar{p}}_{h}^{m+1}$, where the first part $\bar{p}_{h}^{m+1}$, is the solution of the problem

$\frac{\kappa}{\eta} B \bar{p}_{h}^{m+1}=f_{h}^{m+1}, \quad m=0, \ldots, M-1$,

and the second $\overline{\bar{p}}_{h}^{m+1}$ is solution of

$$
\begin{aligned}
& A u_{h}^{m+1}+G \overline{\bar{p}}_{h}^{m+1}=-G \bar{p}_{h}^{m+1}, \\
& \frac{D u_{h}^{m+1}-D u_{h}^{m}}{\tau}+\frac{\kappa}{\eta} B \overline{\bar{p}}_{h}^{m+1}+\frac{h^{2}}{4(\lambda+2 \mu)} B \frac{\overline{\bar{p}}_{h}^{m+1}-\overline{\bar{p}}_{h}^{m}}{\tau} \\
& \quad=-\frac{h^{2}}{4(\lambda+2 \mu)} B \frac{\bar{p}_{h}^{m+1}-\bar{p}_{h}^{m}}{\tau} .
\end{aligned}
$$

We get from (59) and (60)

$$
\begin{gathered}
\tau\left\|\frac{u_{h}^{m+1}-u_{h}^{m}}{\tau}\right\|_{A}^{2}+\left(G\left(\overline{\bar{p}}_{h}^{m+1}-\overline{\bar{p}}_{h}^{m}\right), \frac{u_{h}^{m+1}-u_{h}^{m}}{\tau}\right) \\
=-\left(G\left(\bar{p}_{h}^{m+1}-\bar{p}_{h}^{m}\right), \frac{u_{h}^{m+1}-u_{h}^{m}}{\tau}\right)
\end{gathered}
$$

and

$$
\begin{aligned}
& \left(D \frac{\mathbf{u}_{h}^{m+1}-\mathbf{u}_{h}^{m}}{\tau}, \overline{\bar{p}}_{h}^{m+1}-\overline{\bar{p}}_{h}^{m}\right)+\frac{\kappa}{\eta}\left(B \overline{\bar{p}}_{h}^{m+1}, \overline{\bar{p}}_{h}^{m+1}-\overline{\bar{p}}_{h}^{m}\right) \\
& +\frac{h^{2}}{4(\lambda+2 \mu)}\left(B \frac{\overline{\bar{p}}_{h}^{m+1}-\overline{\bar{p}}_{h}^{m}}{\tau}, \overline{\bar{p}}_{h}^{m+1}-\overline{\bar{p}}_{h}^{m}\right) \\
& =-\frac{h^{2}}{4(\lambda+2 \mu)}\left(B \frac{\bar{p}_{h}^{m+1}-\bar{p}_{h}^{m}}{\tau}, \overline{\bar{p}}_{h}^{m+1}-\overline{\bar{p}}_{h}^{m}\right) .
\end{aligned}
$$

Adding the previous equations and after simple transformations we obtain the inequality

$$
\begin{aligned}
\frac{\kappa}{2 \eta}\left(\left\|\overline{\bar{p}}_{h}^{m+1}\right\|_{B}^{2}-\left\|\overline{\bar{p}}_{h}^{m}\right\|_{B}^{2}\right) \leqslant & \frac{\tau}{2}\left\|G\left(\frac{\bar{p}_{h}^{m+1}-\bar{p}_{h}^{m}}{\tau}\right)\right\|_{A^{-1}}^{2} \\
& +\frac{\tau h^{2}}{8(\lambda+2 \mu)}\left\|\left(\frac{\bar{p}_{h}^{m+1}-\bar{p}_{h}^{m}}{\tau}\right)\right\|^{2} .
\end{aligned}
$$

obtaining the estimate (54).

Convergence results are straightforward using estimates (53) and (54) and considering the approximation errors of the scheme.

\section{Multigrid solution of transformed system}

For the multigrid solution of the Poisson-type operators appearing in Algorithm I in Section 2.3, we can simply choose four times a highly efficient scalar multigrid algorithm that works well for discrete Laplace type operators.
All four scalar operators appearing are isotropic Laplace type operators. A multigrid method with highest efficiency, based on a red-black point-wise Gauss-Seidel smoother, GS-RB, and well-known choices for the remaining multigrid components [12] can be used for all choices of $\lambda, \mu$, and $\tilde{a}$. These include the direct coarse grid discretization of the PDE, full weighting and bilinear interpolation, as the restriction and prolongation operators, respectively.

Remark 2. Here, we give a different view on the algorithm described in Section 2.3. This may be beneficial for analysis purposes. The iterative solution procedure for the transformed system of equations can be interpreted as a "left distributor" for

$\mathbf{C}_{h} \mathbf{L}_{h} \overline{\mathbf{u}}_{h}=\mathbf{C}_{h} \mathbf{f}_{h}$,

with:

$$
\mathbf{C}_{h}=\left(\begin{array}{ccc}
I_{h} & 0 & (\lambda+\mu)\left(\partial_{x}\right)_{h} \\
0 & I_{h} & (\lambda+\mu)\left(\partial_{y}\right)_{h} \\
-\left(\partial_{x}\right)_{h} & -\left(\partial_{y}\right)_{h} & -(\lambda+2 \mu) \Delta_{h}
\end{array}\right) .
$$

In that case, we obtain

$$
\mathbf{C}_{h} \mathbf{L}_{h}=\left(\begin{array}{ccc}
-\mu \Delta_{h} & 0 & L C_{h}^{1,3} \\
0 & -\mu \Delta_{h} & L C_{h}^{2,3} \\
0 & 0 & \tilde{a}(\lambda+2 \mu) \Delta_{h}^{2}-\Delta_{h}
\end{array}\right),
$$

with $L C_{h}^{1,3}=L C_{h}^{3,1}$ and $L C_{h}^{2,3}=L C_{h}^{3,2}$, as in (12), (13), and

$$
\mathbf{C}_{h} \mathbf{f}_{h}=\left(\begin{array}{c}
(\lambda+\mu) \partial_{x} f_{h} \\
(\lambda+\mu) \partial_{y} f_{h} \\
(\lambda+2 \mu) \Delta f_{h}
\end{array}\right)
$$

The main difference with Algorithm I is that here a semidiscretization in time has already taken place (as in model operator (8)). Discrete operator $\mathbf{L}_{h}$ represents the discretization on a collocated grid.

We end up with an upper triangular system. In a first step then, the last equation should be updated after which the other two equations may be treated. For the resemblance and simplification, operator $\tilde{a}(\lambda+2 \mu) \Delta_{h}^{2}-\Delta_{h}$ is split into

$$
\left(-\tilde{a}(\lambda+2 \mu) \Delta_{h}+1\right) q_{h}=\tilde{f}_{h}, \quad-\Delta_{h} p_{h}=q_{h} .
$$

Notice that, compared to the work explained in Section 2.2, we solve the transformed system that is almost (i.e., except for the boundary coupling) decoupled. So, we do not use the transformed system only in multigrid smoothing.

Remark 3. A similar transformation can be defined and analyzed for incompressible Stokes equation from fluid mechanics. In that case, for the discrete Stokes operator

$$
\mathbf{L}_{h, s t}=\left(\begin{array}{ccc}
-\Delta_{h} & 0 & \left(\partial_{x}\right)_{h} \\
0 & -\Delta_{h} & \left(\partial_{y}\right)_{h} \\
\left(\partial_{x}\right)_{h} & \left(\partial_{y}\right)_{h} & 0
\end{array}\right)
$$


a corresponding distributor is given by

$\mathbf{C}_{h, s t}=\left(\begin{array}{ccc}I_{h} & 0 & 0 \\ 0 & I_{h} & 0 \\ -\left(\partial_{x}\right)_{h} & -\left(\partial_{y}\right)_{h} & -\Delta_{h}\end{array}\right)$.

The system then reads

$\mathbf{C}_{h, s t} \mathbf{L}_{h, s t}=\left(\begin{array}{ccc}-\Delta_{h} & 0 & \left(\partial_{x}\right)_{h} \\ 0 & -\Delta_{h} & \left(\partial_{y}\right)_{h} \\ 0 & 0 & -\Delta_{h}\end{array}\right)$.

Also here, it is not necessary to discretize on a staggered grid for a stable discretization. A generalization of this transformation for the system of incompressible NavierStokes equations can be found in [12] (Section 8.8.3).

Assume that $\Omega$ is a bounded domain in $\mathbb{R}^{2}$ and that velocities $u, v$ and pressure $p$ are sufficiently smooth. Then the two systems

$$
\begin{aligned}
& -\Delta u+\operatorname{Re}\left(u u_{x}+v u_{y}\right)+p_{x}=0 \\
& -\Delta v+\operatorname{Re}\left(u v_{x}+v v_{y}\right)+p_{y}=0 \\
& u_{x}+v_{y}=0 \quad(\bar{\Omega}=\Omega \cup \partial \Omega)
\end{aligned}
$$

and

$$
\begin{aligned}
& -\Delta u+\operatorname{Re}\left(v u_{y}-u v_{y}\right)+p_{x}=0 \\
& -\Delta v+\operatorname{Re}\left(u v_{x}-v u_{x}\right)+p_{y}=0 \\
& \Delta p+2 \operatorname{Re}\left(v_{x} u_{y}-u_{x} v_{y}\right)=0 \quad(\Omega) \\
& u_{x}+v_{y}=0 \quad(\partial \Omega)
\end{aligned}
$$

are equivalent. The result originates from [10]. This transformed Navier-Stokes system has recently been used in [11]. Notice that the original primary unknowns are used after the transformation. The generalization to $3 \mathrm{D}$ is trivial.

\section{Numerical experiments}

In this section three numerical experiments are evaluated. The experiments range from a model problem with academic parameter setting to more realistic problems and parameters. We report on the accuracy of the numerical solution from the transformed system and, in particular, on the multigrid convergence. A comparison in terms of CPU time between the staggered multigrid approach and multigrid for the collocated discretization of the transformed system is made.

For the coupled, staggered approach, the measure of convergence is related to the absolute value of the residual after the $m$ th iteration in the maximum norm over the three equations in the system,

$\left|r_{h}^{m}\right|_{\infty}:=\left|r_{1, h}^{m}\right|_{\infty}+\left|r_{2, h}^{m}\right|_{\infty}+\left|r_{3, h}^{m}\right|_{\infty}<T O L$,

with $T O L=10^{-6}$. In the decoupled approach, the stopping criterion is based on the residual in each equation separately. Convergence is achieved in each equation's residual is less than $10^{-6}$.

\subsection{Multigrid convergence for first model problem}

Some analytical reference solutions are known in the literature [1] for (7) in dimensionless form, where scaling has taken place with respect to a characteristic length of the medium $\ell$, Lamé constants $\lambda+2 \mu$, time scale $t_{0}$ and $a(7)$.

By choosing a unit squared domain, a source term $f=2 \cdot \delta_{0.25,0.25} \cdot \sin \hat{t}\left(\delta_{x, y}\right.$ is the Kronecker delta function, $\hat{t}=(\lambda+2 \mu) a t)$, the following boundary and initial conditions,

at: $y=\{0,1\}: \quad u=0, \partial v / \partial y=0$

at: $x=\{0,1\}: \quad v=0, \partial u / \partial x=0$,

and pressure $p=0$ at the boundaries, we can mimic the dimensionless situation. In this case, the solution can be written as an infinite series [1], see also [7]. An interesting feature is that this solution is independent of the Lamé coefficients. The parameters in the reference experiment read $\mu=1 / 2, \lambda=0, \tilde{a}=5 \times 10^{-3}$ (8). Fig. 4 shows for this setting the computed displacement and pressure solution at time $\hat{t}=\pi / 2$. The solution resembles the exact solution in
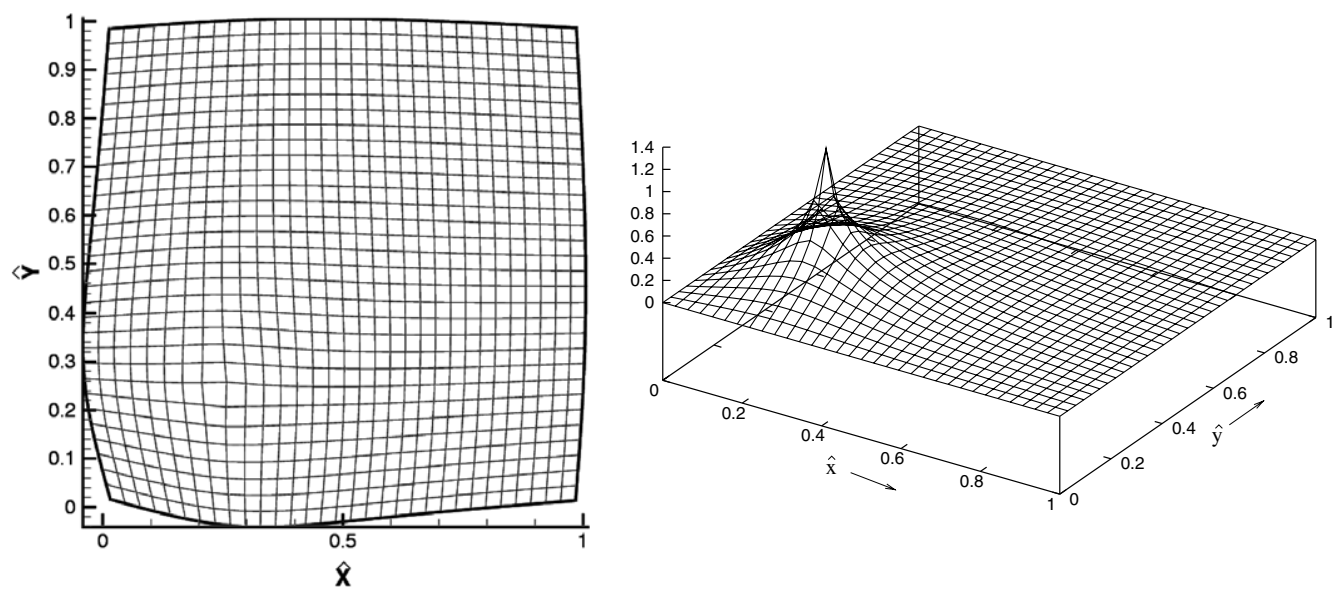

Fig. 4. Numerical solution for displacements and pressure for 2D poroelasticity model problem, $32^{2}$-grid. 
Table 1

CPU times comparison of multigrid methods for the original system on a staggered grid, and the transformed system on a collocated grid

\begin{tabular}{lllll}
\hline & $32 \times 32$ & $64 \times 64$ & $128 \times 128$ & $256 \times 256$ \\
\hline Collocated decoupled & $0.06^{\prime \prime}$ & $0.24^{\prime \prime}$ & $1^{\prime \prime}$ & $4^{\prime \prime}$ \\
Staggered coupled & $1^{\prime \prime}$ & $3^{\prime \prime}$ & $14^{\prime \prime}$ & $55^{\prime \prime}$ \\
\hline
\end{tabular}

[1] very well, without any unphysical oscillations. $\mathrm{O}\left(h^{2}+\tau\right)$ accuracy is observed for the displacements, and, asymptotically, for the pressure too (despite the occurrence of the delta function which usually influences the numerical accuracy negatively) [7].

This reference problem is solved with state-of-the-art multigrid methods developed for the staggered grid (Section 2.2) and the collocated grid discretization (Section 4). Table 1 compares the CPU times, to satisfy the tolerance $10^{-6}$, of the transformed system on the collocated grid and the original system discretized on the staggered grid. Note that this tolerance is considered accurate w.r.t. engineering practice.

The transformed system needs, on average, for the four equations six scalar multigrid iterations per equation. The CPU time in Table 1 for this system on a Pentium IV, $2.6 \mathrm{GHz}$ is $1 \mathrm{~s}$ per time step on a $128^{2}$-grid, and $4 \mathrm{~s}$ per time step on a $256^{2}$-grid. These results are more than 10 times faster than the CPU time results in our previous work with multigrid for the staggered system. The $F(1,1)$-cycle [12] (meaning one pre- and one post-smoothing iteration) is used for this problem; it is fast and shows an $h$-independent behavior. The average multigrid convergence factor observed on the collocated grid is $\approx 0.06$, which is in correspondence with the multigrid theory for Laplace operators. These results not sensitive to variations in the Lamé coefficients.

Also the multigrid convergence factor on the staggered grid is excellent, $\rho_{h} \approx 0.06$. We use the distributive smoother (11) in a multigrid $F(1,1)$-cycle [12] here. In the distributive smoother three operators need to be smoothed separately. For this purpose, a red-black point-wise GaussSeidel smoother is used for the Laplace operators in the first two equations and a line-wise Gauss-Seidel relaxation method for the third equation. This multigrid method for the staggered case was the most efficient method in a comparison among various cycles and smoothers in [7]. The extra costs compared to the collocated version are due to the fact that we treat a coupled system on the staggered grid.

In both solution approaches a matrix-free version of multigrid is used; the CPU times include the time for computing the operator elements.

\subsection{Poroelasticity problem with realistic parameters}

In the following experiment, the domain considered is $\Omega=(-50,50) \times(0,100)$. As the boundary conditions zero displacements are chosen and for the pressure,
Table 2

Material parameters for the second poroelastic problem

\begin{tabular}{lll}
\hline Property & Value & Unit \\
\hline Young's modulus & $3 \times 10^{4}$ & $\mathrm{~N} / \mathrm{m}^{2}$ \\
Poisson's ratio & 0.2 & - \\
Permeability & $10^{-7}$ & $\mathrm{~m}^{2}$ \\
Fluid viscosity & $10^{-3}$ & $\mathrm{~Pa} \mathrm{~s}$ \\
\hline
\end{tabular}

$p= \begin{cases}1 & \text { on } \Gamma_{1}:|x| \leqslant 20, y=100, \\ 0 & \text { on } \Gamma \backslash \Gamma_{1} .\end{cases}$

The material properties of the porous medium are given in Table 2.

In Fig. 5 the solution of the pressure is presented.

In Table 3 we compare the CPU times of the two approaches: Solving the system in a coupled fashion on a staggered grid, and the solution of the decoupled transformed system on a collocated grid. The multigrid methods for both discretization approaches are identical to the ones employed in the previous experiment. The multigrid convergence factor for the transformed system is again excellent, 0.06 for each equation, as is its staggered counterpart $\rho_{h} \approx 0.08$ for the coupled system. The CPU time used, however, differs again substantially, as presented in Table 3.

The results with the transformed system, comparing to the results in Table 1, confirm the independence of the multigrid convergence and CPU time with respect to the poroelastic problem parameters. This is a strong robustness result for the new solver developed.

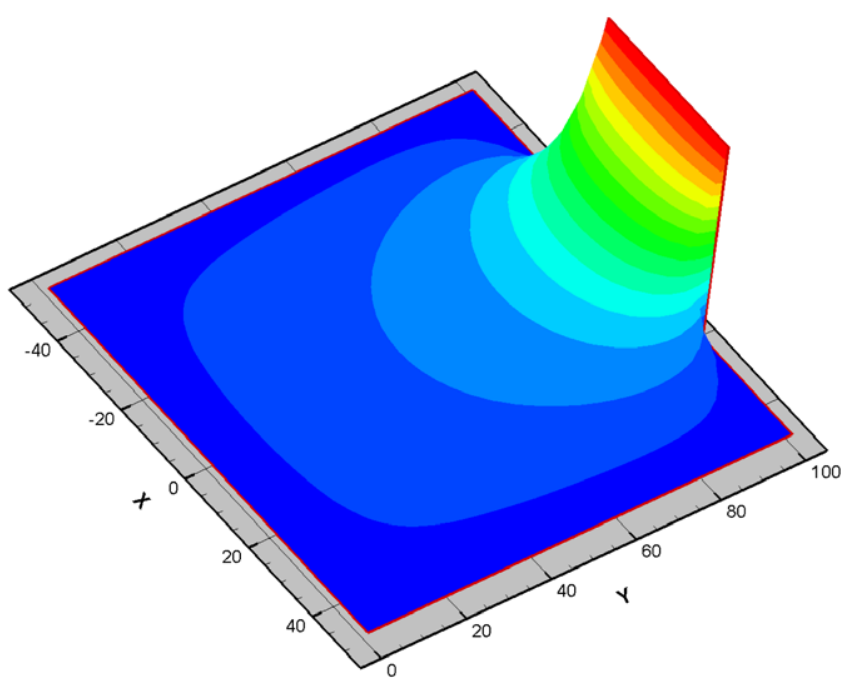

Fig. 5. Numerical solution for pressure for second problem, $32^{2}$-grid.

Table 3

CPU time comparison between the collocated decoupled and the staggered coupled multigrid schemes

\begin{tabular}{lllll}
\hline & $32 \times 32$ & $64 \times 64$ & $128 \times 128$ & $256 \times 256$ \\
\hline Collocated decoupled & $0.05^{\prime \prime}$ & $0.22^{\prime \prime}$ & $1^{\prime \prime}$ & $5^{\prime \prime}$ \\
Staggered coupled & $0.5^{\prime \prime}(5)$ & $2^{\prime \prime}(5)$ & $10^{\prime \prime}(6)$ & $40^{\prime \prime}(6)$ \\
\hline
\end{tabular}




\subsection{Poroelastic footing experiment}

The third example is a true $2 \mathrm{~d}$ footing problem (see also [9]). The simulation domain is a 100 by 100 meters block of porous soil, as in Fig. 6.

At the base of this domain the soil is assumed to be fixed while at some upper part of the domain a uniform load of intensity $\sigma_{0}$ is applied in a strip of length $40 \mathrm{~m}$. The whole domain is assumed free to drain. Therefore, the boundary data are given as follows:

$p=0, \quad$ on $\partial \Omega$,

$\sigma_{x y}=0, \quad \sigma_{y y}=-\sigma_{0}$,

on $\Gamma_{1}=\{(x, y) \in \partial \Omega, /|x| \leqslant 20, y=100\}$,

$\sigma_{x y}=0, \quad \sigma_{y y}=0$,

on $\Gamma_{2}=\{(x, y) \in \partial \Omega, /|x|>20, y=100\}$,

$\mathbf{u}=\mathbf{0}, \quad$ on $\partial \Omega \backslash\left(\Gamma_{1} \cup \Gamma_{2}\right)$.

The material properties of the porous medium are the same as in the previous problem, see Table 2 and the uniform load is taken as $\sigma_{0}=1 \times 10^{4} \mathrm{~N} / \mathrm{m}^{2}$.

Notice that the boundary condition for the footing problem involves the prescription of stress conditions. These conditions are applied in the discretization of the equations of the stabilized system $\left(\left(\mathbf{P}^{\text {tr }}\right)_{\mathbf{h}}\right)^{-\mathbf{t r}}$. Then they are transformed to problem $\left(\mathbf{P}^{\mathbf{t r}}\right)_{\mathbf{h}}$ in a similar way as it explained in Section 3.2.

In Fig. 7 the solution of the pressure is presented. The unphysical oscillations for small $t$ that were present in the numerical results in [9], do not occur here with both formulations: not with staggered grids and not with collocated grids adding the stabilization term.

Finally, the multigrid convergence factor for the decoupled system is found to be 0.06 for the equations for $p$ and $q$, while for the other two equations, with the stress boundary conditions, it is found to be 0.12 . The corresponding CPU times are $1^{\prime \prime}$ on a $128^{2}$-grid and $4^{\prime \prime}$ on a $256^{2}$-grid. The convergence factor for the coupled system on the stag-

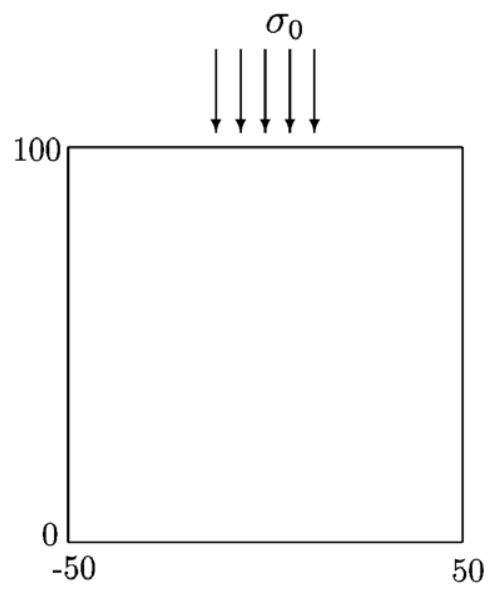

Fig. 6. Computational domain for the footing problem

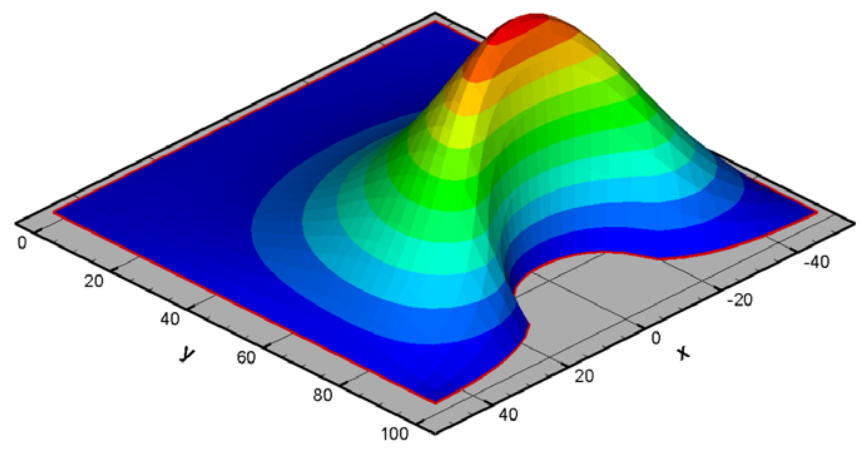

Fig. 7. Numerical solution for displacements and pressure for $2 \mathrm{D}$ poroelasticity reference problem, $32^{2}$-grid.

gered grid reads $\rho_{h}=0.2$. The CPU times are then $29^{\prime \prime}$ on a $128^{2}$-grid and $113^{\prime \prime}$ on the $256^{2}$-grid. The improvement in CPU time with the transformed system is here more impressive. This example shows that the transformation can easily be applied to more realistic boundary conditions, resulting in a similar performance of the solver.

\section{Conclusion}

In this paper we provide a fast and accurate discrete solution for the incompressible variant of the poroelasticity equations. The system is transformed so that a stable discretization can be obtained on a collocated grid. This is done by means of an (implicit, via the transformation) addition of an $\mathrm{O}\left(h^{2}+\tau\right)$ stabilization term in the discretization of the transformed system.

A robust and very efficient multigrid iteration has been defined based on the decoupled version of the poroelasticity system after the transformation. It is sufficient to choose a highly efficient multigrid method for a scalar Poisson type equation for the overall solution of this poroelasticity system. With standard geometric transfer operators, a direct coarse grid discretization and a point-wise red-black Gauss-Seidel smoother, an efficient multigrid method is developed for all relevant choices of the problem parameters. According to classical multigrid theory, we observe multigrid convergence factors that are less than 0.1 for a variety of poroelastic problems. In the literature this is often called "textbook multigrid efficiency". Very satisfactory solution times have been produced, that are about 10 times faster than the iterative solution of the coupled system, discretized on a staggered grid.

The present discretization and iterative solution method can be seen as a basis for the generalization to more complicated problems that are also both porous and elastic. Here we think of double porosity problems, or coupled problems in which poroelasticity is coupled to Stokes flow and to thermodynamical models.

The present saddle point type problem has been handled well by a transformation, that is now well understood, at least in 1D. The future problems offer next challenges in the treatment of coupled saddle point type problems. 


\section{References}

[1] S.I. Barry, G.N. Mercer, Exact solutions for 2D time dependent flow and deformation within a poroelastic medium, ASME J. Appl. Mech. 66 (1999) 536-540.

[2] M. Benzi, G.H. Golub, J. Liesen, Numerical solution of saddle point problems, Acta Numer. (2005) 1-137.

[3] M.A. Biot, General theory of three dimensional consolidation, J. Appl. Phys. 12 (1941) 155-164.

[4] A. Brandt, Multigrid techniques: 1984 guide with applications to fluid dynamics, GMD-Studie Nr. 85, Sankt Augustin, Germany, 1984.

[5] W. Ehlers, J. Blum (Eds.), Porous Media: Theory, Experiments and Numerical Applications, Springer, Berlin, Germany, 2002.

[6] F.J. Gaspar, F.J. Lisbona, P.N. Vabishchevich, A finite difference analysis of Biot's consolidation model, Appl. Numer. Math. 44 (2003) 487-506.

[7] F.J. Gaspar, F.J. Lisbona, C.W. Oosterlee, R. Wienands, A systematic comparison of coupled and distributive smoothing in multigrid for the poroelasticity system, Numer. Linear Algebra Appl. 11 (2004) 93-113.
[8] V.C. Mow, W.M. Lai, Recent developments in synovial joint biomechanics, SIAM Rev. 22 (1980) 275-317.

[9] M.A. Murad, A.F.D. Loula, On stability and convergence of finite element approximations of Biot's consolidation problem, Int. J. Numer. Methods Engrg. 37 (1994) 645-667.

[10] A. Schüller, A multigrid algorithm for the incompressible NavierStokes equations, in: W. Hackbusch, R. Rannacher (Eds.), Numerical Treatment of Navier-Stokes Equations, Notes on Numerical Fluid Mechanics, vol. 30, Vieweg, Braunschweig, 1990, pp. 124-133.

[11] R.C. Swanson, Evaluation of a multigrid scheme for the incompressible Navier-Stokes equations, NASA/TM-2004-213026, May, 2004.

[12] U. Trottenberg, C.W. Oosterlee, A. Schüller, Multigrid, Academic Press, New York, 2001.

[13] R. Wienands, F.J. Gaspar, F.J. Lisbona, C.W. Oosterlee, An efficient multigrid solver based on distributive smoothing for poroelasticity equations, Computing 73 (2004) 99-119. 